Benchmark Imagery Project,

Report on Generation of

Synthetic Images

J. Goforth, T. White, P. Pope, R. Roberts, I.

Burns, L. Gaines

October 11, 2012 
This document was prepared as an account of work sponsored by an agency of the United States government. Neither the United States government nor Lawrence Livermore National Security, LLC, nor any of their employees makes any warranty, expressed or implied, or assumes any legal liability or responsibility for the accuracy, completeness, or usefulness of any information, apparatus, product, or process disclosed, or represents that its use would not infringe privately owned rights. Reference herein to any specific commercial product, process, or service by trade name, trademark, manufacturer, or otherwise does not necessarily constitute or imply its endorsement, recommendation, or favoring by the United States government or Lawrence Livermore National Security, LLC. The views and opinions of authors expressed herein do not necessarily state or reflect those of the United States government or Lawrence Livermore National Security, LLC, and shall not be used for advertising or product endorsement purposes.

This work performed under the auspices of the U.S. Department of Energy by Lawrence Livermore National Laboratory under Contract DE-AC52-07NA27344. 


\title{
Benchmark Imagery Project Report on Generation of Synthetic Images
}

\author{
John W. Goforth ${ }^{1}$, W. Travis White III ${ }^{1}$, Paul A. Pope ${ }^{2}$, \\ Randy S. Roberts ${ }^{1}$, lan G. Burns ${ }^{2}$, Lucinda R. Gaines ${ }^{2}$ \\ ${ }^{1}$ Lawrence Livermore National Laboratory \\ ${ }^{2}$ Los Alamos National Laboratory
}

September 27, 2012

\section{BACKGROUND}

The "Benchmark Imagery" project is a DOE NA-22-funded effort whose three-year goal is to create a suite of imagery that can be used to test geospatial algorithms used for extracting semantic content from overhead images of industrial facilities (e.g., auto-annotation). The project is a collaborative effort between Los Alamos National Laboratory (LANL) and the Lawrence Livermore National Laboratory (LLNL). Although a variety of image modalities are used to capture overhead photographs of terrestrial facilities, the current focus of the effort of this project is on collecting and annotating conventional visual-band (RGB or RGB/NIR) images.

Presently, the set of images consists of 114 airborne images of various US industrial facilities. We have annotated all of the images in the imagery set, using a constrained vocabulary of about a dozen general terms such as "building," "tower," or "electrical substation." The project plans to include synthetic and composite images in addition to actual photographs. In preparation for using synthetic images, we have built a proof-of-principle synthetic image of a fictitious nuclear power plant. This report describes the key aspects of creating that image.

\section{MOTIVATION}

Synthetic images have numerous advantages over real satellite images. First and foremost, the content in a synthetic image is entirely configurable. Creating facilities, objects, and environment in a synthetic image can be dictated by the end user and their needs. This is very important when testing algorithms on situations where imagery is limited. Facilities can be generated in different stages of construction. Images can be "captured" at different angles and different time of day. Effects such as fog, clouds, and lighting can be simulated. Sensor and mission characteristics such as over-exposure, distortion, blur, flight patterns can be modeled. Synthetic image generation is not limited to red-greenblue (RGB) visual-band imagery. Infrared and hyperspectral images are also possible to generate synthetically. LIDAR and Shapefiles of 3D geometry can be generated as well. 
Synthetic images are also more distributable than real images because they tend to be less sensitive in nature (unclassified). Since a site may be fictional, it is easier to distribute to universities and other non-governmental entities.

For our first synthetic scene we have modeled a nuclear power plant. This decision was made because of several factors:

- Nuclear power plants are of interest to NA-22.

- Subject Matter Experts (SMEs) in nuclear power plant facilities are readily available at both LLNL and LANL.

- High quality synthetic 3D models of nuclear power components are easy and low-cost to acquire commercially.

- Some algorithm developers have expressed interested and/or current work in automatically identifying these facilities.

\section{THE SYNTHETIC IMAGE}

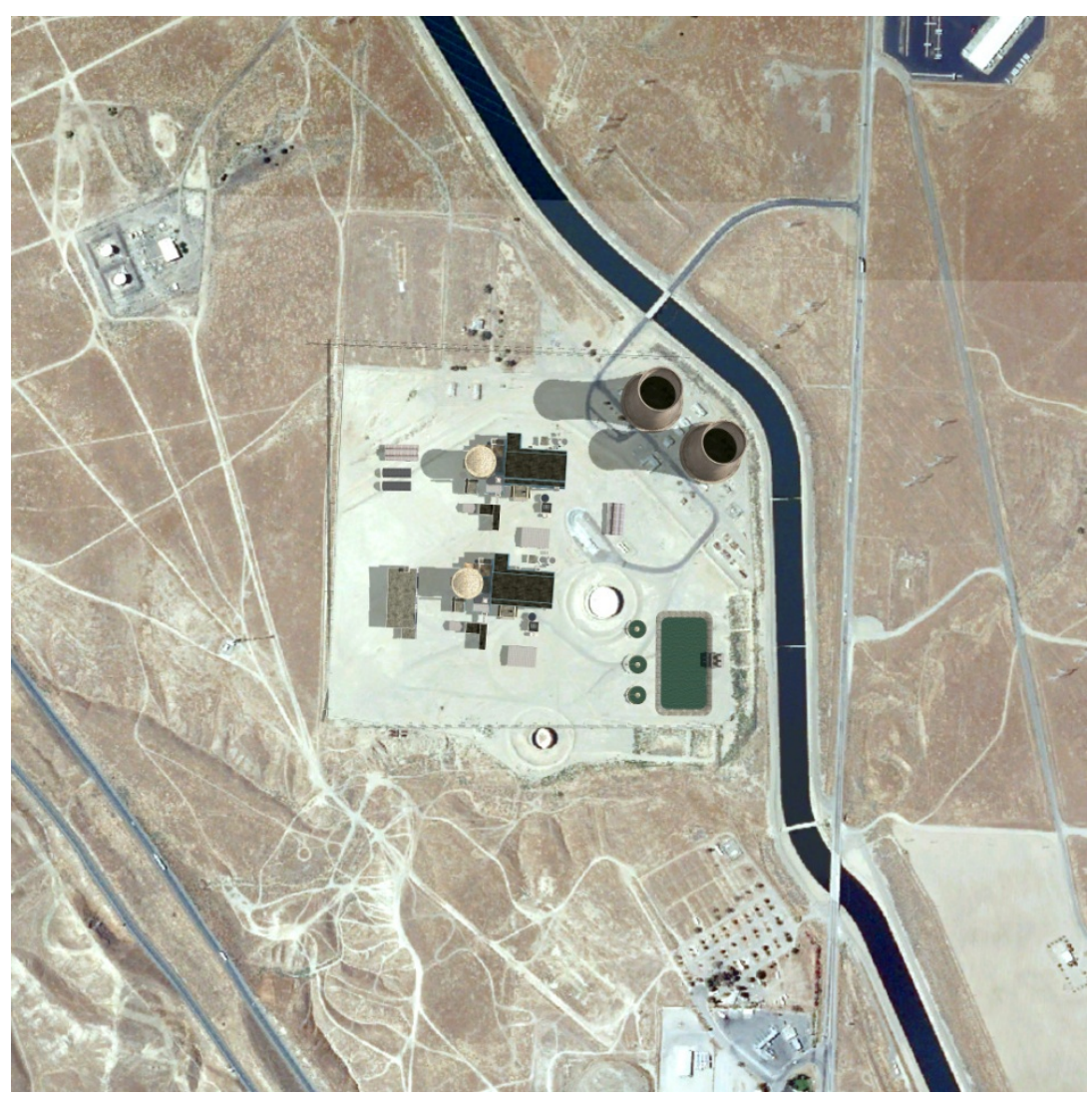

Figure 1 - The Synthetic Image 
The synthetic image above we have generated as our milestone deliverable. It combines real satellite imagery, real elevation data, and synthetic 3D models with textures. The 3D models, textures, and layout of the facility are intended to be illustrative of a nuclear power plant, not a rigorously vetted model. The image is based on images of nuclear power plants collected in the Benchmark Imagery suite and discussions with subject-matter experts. Future work will refine the accuracy of 3D models, textures and layout. There are also synthetic lighting, shadows, and post-processing color correction. The camera view is directly overhead. There are 2048 by 2048 pixels with a ground sampling distance (GSD) of 0.79 meters/pixel. There is no cloud cover or fog, because this image is intended to be tested with algorithms that cannot yet handle obscuration.

The components of the facility are synthetic textured 3D models that are commercially available from TurboSquid 3D model marketplace[6]. There are 8 unique components types, and 16 total components.

\section{Components in Synthetic Image:}

- Reactor with connected buildings (2)

- Cooling Tower (2)

- Large Pool (1)

- Small Pool (3)

- Fence (3)

- Office Building (1)

- Building A (2)

- $\quad$ Building B (2)

The "canvas" or base terrain is made up of real satellite imagery that has been "draped over" elevation data in a 3D world. The elevation data and satellite imagery are for Kettleman City, CA (Lat/Lon 35.996N/119.96W.) The site was selected in part because there was a large empty area that would provide a suitable "canvas" for placing components. There is also a water source in the area which is required by Nuclear Power Plants (although sometimes out of view and utilized through underground pipes).

Some components/features which will be added in future iterations are missing from the proofof-principle image. These include parking lots, vehicles, containers, logistical equipment, electrical substation/switchyard, and many more objects that are typically found in a nuclear power plant.

The image has been generated in the visual (EO) band, because that is consistent with the images that we have gathered in the benchmark imagery set.

The lighting/shadows are based on a date and time of August $8^{\text {th }}, 2011,0800$ PST. Each model has textures which define material properties that describe how lighting interacts with the textures. These material properties were tuned to create lighting of models that correctly matches the based imagery to increase realism. 
The synthetic image has embedded geospatial information in GeoTIFF format.[1] This information is required by most geospatial analysis applications such as GlobalMapper.[2] It allows such applications and algorithms to calculate distances and terrestrial locations of objects in the image.

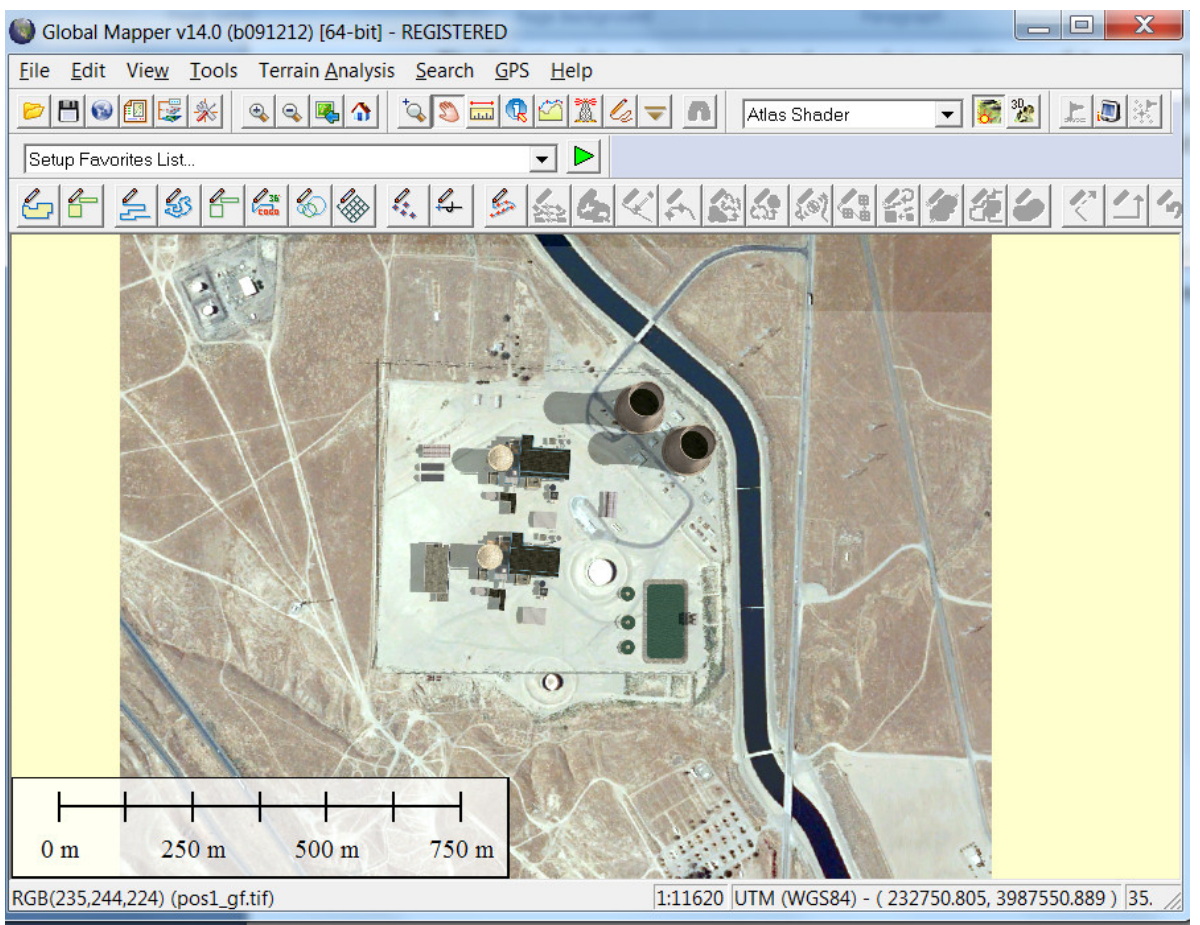

Figure 2 - Synthetic image loaded in GlobalMapper GIS software which displays UTM coordinates and a distance scale in meters.

\section{IMAGE CREATION}

The following images (figures 3-5) highlight the detail of the 3D scene and show additional features such as cloud cover that will be used in future synthetic images. These images are for demonstration purposes and are not intended to be used for algorithm testing, because they do not match the parameters of the benchmark imagery set and/or typical ground imagery (e.g, GSD, lookdown angle or cloud cover) . 


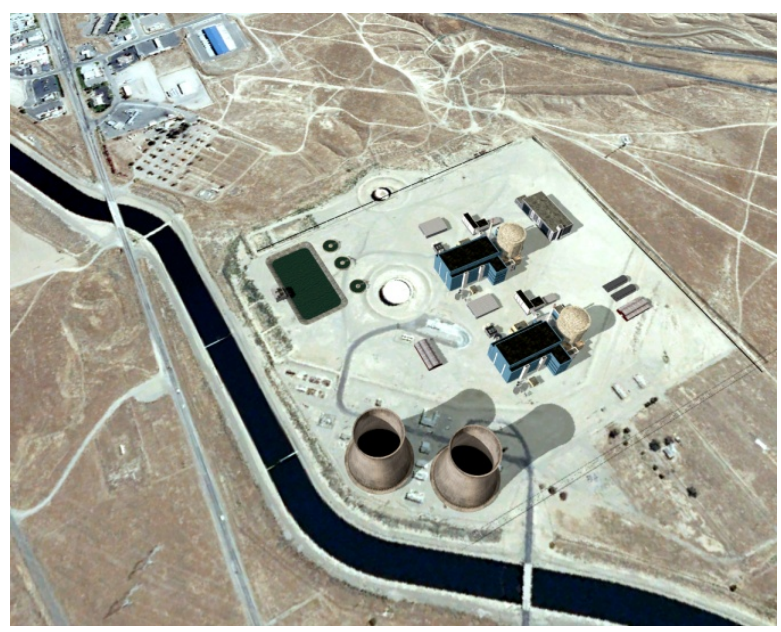

Figure 3 - Oblique View of Synthetic Scene

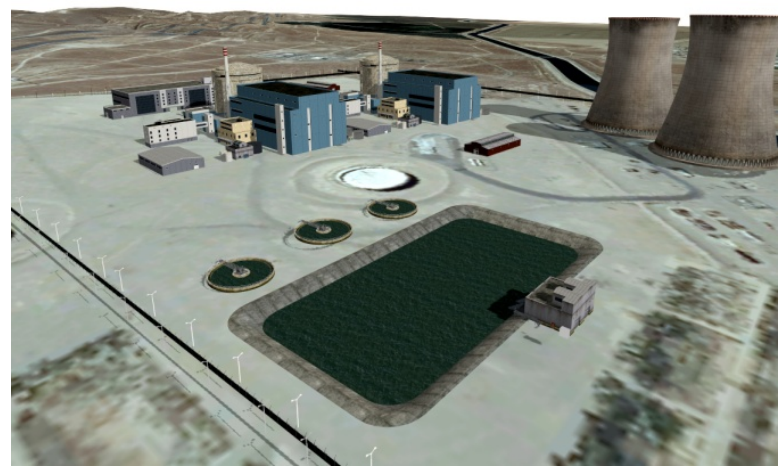

Figure 4 - Close-up view of synthetic facility to highlight model detail.

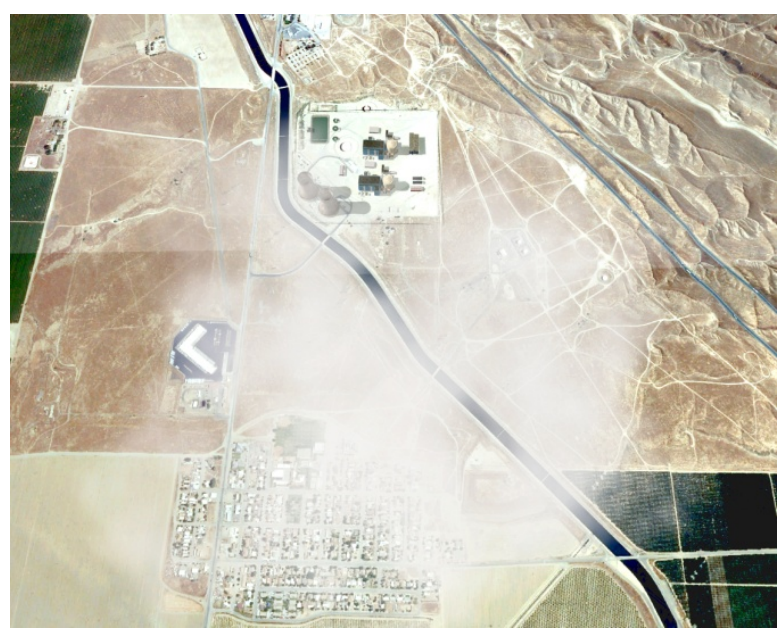

Figure 5 - Example of Cloud Obscuration 


\section{Approach}

To create synthetic images we used the SceneWorks software, a real-time 3D visualization framework developed by LLNL. It has over 6 years of development and is used by the Army (as "ACATS 3D"),[3] Navy (as "JECP SPM 3D"),[4] Air Force, NGA, and DHS. Real-time 3D render software, such as SceneWorks, leverages the video-game industry and the related advances in off-the-shelf video cards. Images take approximately $1 / 30^{\text {th }}$ of a second to be generated, allowing for real-time 3D interaction. Real-time interaction is a tremendous benefit when setting up the scene, placing components, tweaking lighting/materials, etc.

To generate the synthetic image, we first captured the image in SceneWorks using the "Save Screenshot" feature in the video recorder plug-in. The image was then post-processed using "Gimp" (an open source Photoshop-like tool.) The post processing effects we used are listed below under "SceneWorks 3D Generation Details". The post-processing allowed us to enhance the realism of the image by more closely matching the colors and exposure of the images that are in the benchmark imagery set. This was done visually by hand and did not model the actual characteristics of any particular sensor used to gather real images in the benchmark imagery set.

After post-processing, the UTM coordinates of the four corners of the image were acquired using SceneWorks' GIS capabilities. To generate a GeoTIFF image from the post-processed TIFF image, the geospatial data (GSD, UTM extents) were added to the TIFF file as GeoTIFF metadata using "listgeo" and "geotifcp" tools. [4]

\section{SceneWorks 3D Generation Details}

\begin{tabular}{|c|c|}
\hline Rendering & $\begin{array}{l}\text { Video Card: } \\
\text { - NVIDIA Quadro 3000M (laptop) } \\
\text { Video Rendering Specs: } \\
\text { - } 16 X \text { Anisotropic Filtering } \\
\text { - } 32 X \text { Anti-Aliasing } \\
\text { - } 8 X \text { Anti-Aliasing Transparency Super-sampling }\end{array}$ \\
\hline Post processing & $\begin{array}{ll}\text { - } & \text { Enhanced Brightness } \\
\text { - } & \text { Enhanced Contrast } \\
\text { - } & \text { Enhanced Saturation } \\
\text { - } & \text { Enhanced Lightness }\end{array}$ \\
\hline
\end{tabular}

\section{Alternate Approaches Considered}

We considered alternate approaches, including DIRSIG (developed by RIT) which is a ray tracing application. Ray tracing (as compared to 3D real-time) is a physics-like simulation of photons to generate an image. It is more physically based than 3D real-time approaches, which can result in better 
quality atmospherics, reflectivity, and non-EO visible (IR / hyper spectral) images. A drawback to ray tracing (physics-based models) is that with our current hardware (off-the-shelf laptop) a single frame can take over 20 hours of CPU time to generate. Because we are still in an exploratory phase of generating synthetic images for use in the development and validation of semantic extraction algorithms, it is not clear whether having detailed physics-based models is more important than being able to create a wide variety of scenes quickly and easily. As an initial approach, we have chosen to use SceneWorks and off-the shelf 3D models of scene components in order to create a larger set of scenes more quickly. We will reconsider other approaches in the future.

\section{CONCLUSION \& PATH FORWARD}

We have successfully generated a synthetic image of a nuclear power plant that can be used by algorithm developers. The image is consistent with the photographic images in our benchmark imagery set in both appearance and geospatial embedded information.

In our FY13 work we will be generating more synthetic images and working with algorithm developers to understand their needs and the results of using our synthetic imagery. Planned work includes:

- Systematically building up scenes with increasing clutter and complexity

- Enhancing the realism of the Nuclear power plant (parking lots, vehicles, containers, logistical equipment, electrical substation/switchyard, etc.)

- Creating different configurations of the same facility (nuclear power plant)

- Generating images of a single facility at different stages of construction/lifecycle.

- Generating images of different types of facilities (e.g. chemical processing, heavy manufacturing, etc.)

\section{References}

1. http://www.remotesensing.org/geotiff/faq.html

2. Blue Marble GlobalMapper software http://www.bluemarblegeo.com/global-mapper/

3. "Predicting Pollutant Dispersion and Exposure in Mixed Outdoor-Indoor-Vehicle Environments" Jeffery Peterson, US Army Dugway Proving Ground, http://cbdstconf2010.sainc.com/parallelsessionview2.aspx?conferencesessionid=22

4. "Joint Expeditionary Collective Protection System Performance Model (JECP SPM) Overview" Gaurang Dave, Naval Surface Warfare Center, Dahlgren, http://cbdstconf2010. sainc.com/parallelsessionview2.aspx?conferencesessionid=22

5. http://www.remotesensing.org/geotiff/geotiff.html

6. http://www.turbosquid.com 